\title{
Solar and atmospheric neutrino oscillations in Super-Kamiokande
}

\author{
Yusuke Koshio*t \\ Okayama university \\ E-mail: koshiodokayama-u.ac.jp
}

\begin{abstract}
The recent results of solar and atmospheric neutrino oscillations in Super-Kamiokande (SK) are presented. SK has four phases (SK-I to IV) since starting the operation in 1996. In this time update, the 2365 and 2520 days of data for SK-IV was used for solar and atmospheric neutrino analysis, respectively. In solar neutrino analysis, the lowering energy threshold (3.5MeV) has achieved in SK-IV thanks to the background reduction. More than 84,000 signals were totally observed in 5200 days through all the phases, and no long term flux time variation was seen. The night flux was 3.3\% higher than day flux which caused by the neutrino oscillation of the matter effect of the earth at 3 sigma level. No significant distortion of the energy spectrum was seen. The obtained global neutrino oscillation parameters were determined as $\Delta m_{21}^{2}=7.49 \times 10^{-5} \mathrm{eV}^{2}$ and $\sin ^{2} \theta_{12}=0.308$. In atmospheric neutrinos, 0.33 MegatonYears of data were accumulated, and the analysis were carried out. The best fit of the neutrino oscillation parameters are $\Delta m_{32}^{2}=$ $2.5 \times 10^{-3}, \sin ^{2} \theta_{23}=0.587, \delta_{C P}=4.189$ for normal hierarchy case. The normal hierarchy is preferred with significance $\Delta \chi^{2}=\chi^{2}(N H)-\chi^{2}(I H)=-4.3$. The tau neutrino appearance by atmospheric neutrino oscillation was confirmed at $4.6 \sigma$ level.
\end{abstract}

Neutrino Oscillation Workshop

4 - 11 September, 2016

Otranto (Lecce, Italy)

${ }^{*}$ Speaker.

${ }^{\dagger}$ for Super-Kamiokande collaboration 


\section{Super-Kamiokande}

The Super-Kamiokande detector (SK) is a cylindrical tank $(39.3 \mathrm{~m}$ in diameter and $41.4 \mathrm{~m}$ in height) filled with 50 kilotons of pure water. It is located $1000 \mathrm{~m}$ underground $(2,700 \mathrm{~m}$ water equivalent) in the Kamioka mine in Gifu Prefecture, Japan. The detector is divided two region called "inner" and "outer", and lined with 11,129 20-inch PMTs in the inner detector and 1,885 8inch PMTs in the outer detector, respectively. The signal detection method in SK is that Cherenkov light generated in water from charged particles are observed by the PMTs. [W] The fiducial volume used for the data analysis is 22.5 kilotons, which is within the $2 \mathrm{~m}$ from the inner wall, to keep the detector performance good. SK started in 1996, and there are four experimental phases. The latest SK-IV started in 2008 after new electronics (QBEE []]) was installed. Due to this new electronics, it makes the detection efficiency of decay electron from stopping muon higher. It is also possible to tag a $2.2 \mathrm{MeV}$ gamma-ray which is produced by the neutron capture by proton in water even though it is about $20 \%$ detection efficiency. The detector is well calibrated at less than $1 \%$ level for its energy scale, position determination, and so on[B]].

\section{Solar neutrinos}

Solar neutrinos are generated by nuclear fusion reaction via pp-chain and CNO cycle deep inside the sun. Among several kinds of solar neutrinos, SK can mainly detect boron- 8 neutrinos $\left({ }^{8} \mathrm{~B}\right)$ which is high energy component (the end point is about $16 \mathrm{MeV}$ ) because of the energy threshold. Due to its large fiducial mass (22.5 kiloton for above $5.5 \mathrm{MeV}, 13.3$ kiloton for $4.5 \sim 5.5 \mathrm{MeV}, 8.8$ kiloton for 3.5 4.5MeV), SK gives the most precise measurement of the ${ }^{8} \mathrm{~B}$ solar neutrino flux. SK detects solar neutrinos through neutrino-electron elastic scattering, $v+e \rightarrow v+e$, whose energy, direction and time of the recoil electron can be measured. Since the electron from this reaction preserve the original direction of neutrino, the solar direction can be seen. The SK detector performance for solar neutrino detection is that the vertex resolution is $55 \mathrm{~cm}$, angular resolution is 23 degree, and the energy resolution is $14 \%$ at $10 \mathrm{MeV}$ electron. In addition to the above calibration, the calibration by LINAC and DT are performed mainly for solar neutrino analysis, and achieved the energy determination within $0.5 \%$ precision. [四, [] The motivation of the observation in SK is mainly seeing directly the neutrino oscillation MSW effect, especially a solar neutrino spectrum distortion induced by the matter of the sun and a day-night flux asymmetry by the earth. Assuming the current observed neutrino oscillation parameters, a distortion of the solar neutrino energy spectrum is expected. Indeed, it is expected to increase with decreasing energy (the "up-turn" effect) in a few $\mathrm{MeV}$ energy region due to the transition from matter dominated oscillations to vacuum oscillations. It is also expected to have $2-4 \%$ level of day-night flux asymmetry.

The total live time of the solar neutrino analysis in this time is 5200 days. Thanks to the lowering trigger threshold is started from May 2015, the detection efficiency in the lowest energy bin, 3.5 4.0 MeV in kinetic energy of recoil electrons, is more than $99 \%$ comparing to the previous setting, 84\%. Since the direction of recoil electron observed in SK preserve the original incident neutrino direction, clear peak to the solar direction in the final sample can be seen as shown in

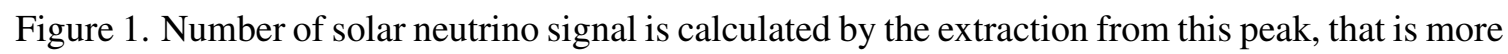
than 84000 , and observed ${ }^{8} \mathrm{~B}$ neutrino flux is $2.355 \pm 0.033 \times 10^{6} \mathrm{~cm}^{-2} \mathrm{~s}^{-1}$. The flux systematic 
uncertainty in SK-IV is $1.7 \%$, compared to $3.2-3.5 \%$ for SK-I and $2.1 \%$ for SK-III. The sources of this reduced uncertainty are from trigger efficiency, vertex shift and angular resolution. The Figure $\square$ shows the observed ${ }^{8} \mathrm{~B}$ solar neutrino rate as a function of time overlaid with the sun spot number. The solar neutrino rate is consistent with constant, its reduced chi-square is $15.52 / 19$ (d.o.f.).

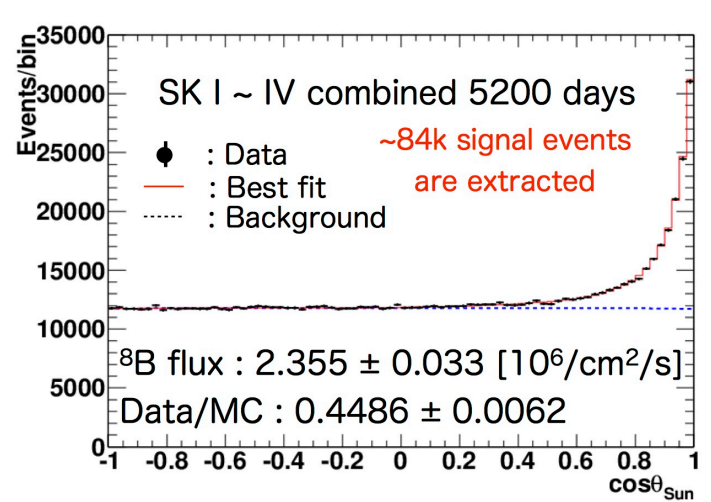

Figure 1: The solar neutrino signal combined to SK-I to SK-IV.

The day-night flux difference is observed, and the calculation method is described in [焑]. The combined result from SK-I to SK-IV is $-3.3 \pm 1.0 \pm 0.5 \%$ assuming neutrino oscillation parameters fitting by solar neutrino measurements. The significance of non-zero day-night asymmetry is $3.0 \sigma$. The day-night asymmetry mainly depends on $\Delta m_{21}^{2}$ as shown in Figure [3. The observed asymmetry, shown as hatched regions with center line, are consistent with the expectation from the current neutrino oscillation parameters which are shown as the vertical hatched regions at 1.0 to $1.3 \sigma$ level.

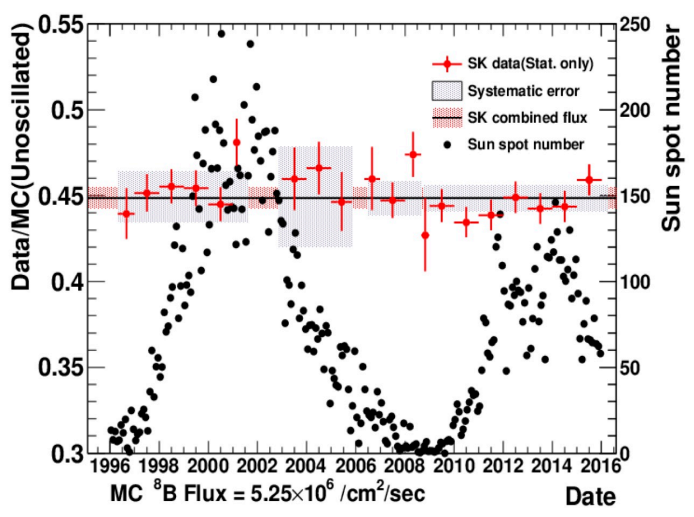

Figure 2: The observed solar neutrino flux decided by the expected flux with the sun spot number as a function of time.

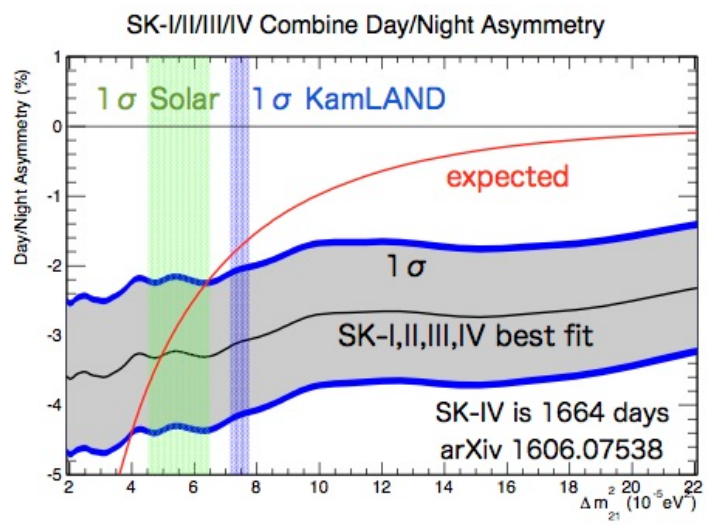

Figure 3: Day-night asymmetry as a function of $\Delta m_{21}^{2}$. The gray hatch show the measurement with $1 \sigma$ statistical uncertainties and additional blue band shows the inclusion of the systematic uncertainties.

No distortion in the recoil electron energy spectrum is not be found in all the SK phases. The neutrino energy spectrum shape analysis is also performed. In SK, unfortunately, only the recoil electron energy is observed, and the original neutrino energy cannot be reconstructed. However, it is possible to derive the neutrino energy spectrum by de-convolution of the observed recoil electron spectrum in SK. In order to get it, the polynomial function shown in Figure $\mathbb{\text { G }}$ was introduced as a survival probability. Figure $\$$ shows the allowed region of $v_{e}$ survival probability as a function of neutrino energy. The result is consistent with MSW prediction at one sigma level. 
Neutrino oscillation analysis in SK was done by comparison between the observed and predicted spectrum and time variation of all the phases. The best fit parameters $\operatorname{are~}^{2} \sin ^{2} \theta_{12}(S K)=$ $0.337_{-0.023}^{+0.027}, \Delta m_{21}^{2}(S K)=4.74_{-0.80}^{+1.40} \times 10^{-5} \mathrm{eV}^{2}$. This result has about $2 \sigma$ tension with the KamLAND reactor result for $\Delta m_{21}^{2}\left(7.54_{-0.17}^{+0.19} \times 10^{-5} \mathrm{eV}^{2}\right)$. Figure $\square$ shows the allowed region from global solar neutrino data, which includes SK, SNO, Radiochemical and Borexino, overlaid with

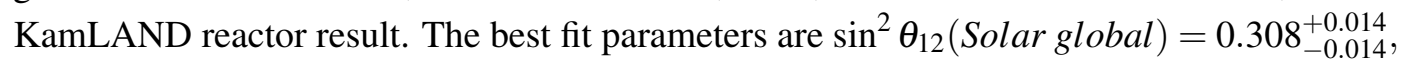
$\Delta m_{21}^{2}($ Solar global $)=4.84_{-0.60}^{+1.26} \times 10^{-5} \mathrm{eV}^{2}$.

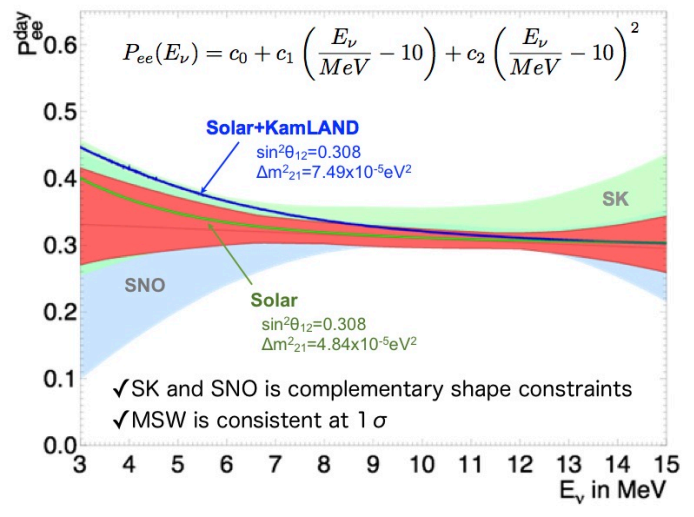

Figure 4: Allowed region of the $v_{e}$ survival probability obtained by SK (green), SNO (blue), and Solar global (red), overlaid with the expected curve from the neutrino oscillation parameters.

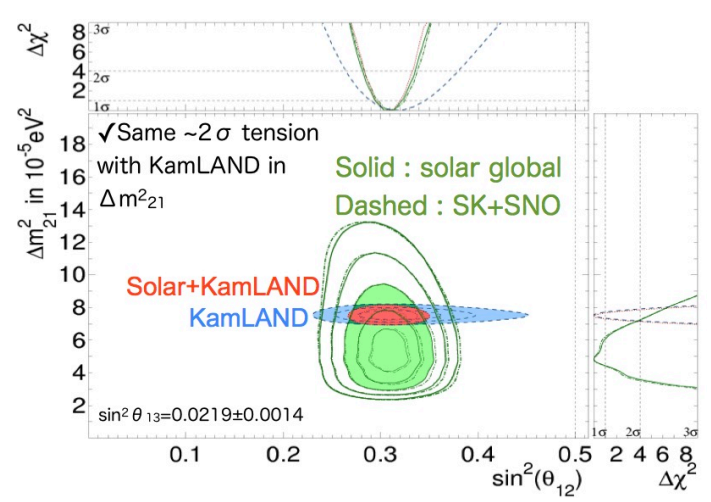

Figure 5: Neutrino oscillation parameter allowed region from all the solar neutrino experiments (green), KamLAND (blue) and Solar+KamLAND (red) from 1 to 5 sigma lines and 3 sigma area.

\section{Atmospheric neutrinos}

Atmospheric neutrinos are generated when primary cosmic rays strike air nuclei and the outgoing hadron decays. The flux is measured by SK[D], and the model calculation [ [8] is consistent with data. In the observation in SK, the data sample is categorized by three distinct topologies: fully-contained (FC), partially-contained (PC), and upward-going muon (UPMU). FC events have reconstructed interaction vertices inside the fiducial volume, while PC events also have interaction vertices within the fiducial volume of the inner detector, but there is significant light in the outer detector volume. FC is further divided to single and multi ring, and electron-like and muon-like events. UPMU events are the highest-energy events in the SK detector and are due to muon neutrino interactions in the surrounding rock that produce penetrating muons. These muons either stop in the inner detector volume (stopping events) or continue through the inner detector (through-going events).

For three flavor neutrino oscillation analysis using atmospheric neutrinos in SK, all the subleading effects due to $\theta_{13}$ and $\Delta m_{21}^{2}$ is considered. Here, the earth matter effect plays an important role in these neutrino oscillation schemes, e.g. the effect on $\theta_{13}$ resolves mass hierarchy, while $\Delta m_{21}^{2}$ resolves octant $\theta 23$. It has different behavior between $v_{e}$ and $\bar{v}_{e}$ for the mass hierarchy. In 
SK, they are statistically separated based on the number of decay electrons, number of cherenkov rings, and the transvers momentum.

The total live time of the atmospheric neutrino analysis in this time is 0.33 MtonYear, and the PMT gain correction, which is continuously increasing, is newly applied. Newly updated Monte Carlo simulations are also applied, those are Honda 11[8] for atmospheric neutrino generation, NEUT for neutrino interaction, and detector simulation. Figure 6 shows the momentum and zenith angle distribution in each data category. Using all these data, neutrino oscillation analysis was performed. Figure $\square$ shows the chi-square differences from the minimum as a function of $\Delta m_{32}^{2}$ (or $\left.\Delta m_{13}^{2}\right), \sin ^{2} \theta_{23}$ and $\delta_{C P}$, for the normal and inverted hierarchy cases. The best fit parameters are $\Delta m_{32}^{2}=2.5 \times 10^{-3}, \sin ^{2} \theta_{23}=0.587, \delta_{C P}=4.189$ for normal hierarchy case. The p-value for obtaining a result favoring the normal hierarchy as much as the observed data is $0.45(0.03)$ assuming a normal (inverted) hierarchy and $\sin ^{2} \theta_{23}=0.6$. If the $\mathrm{T} 2 \mathrm{~K}$ results are combined, the tendency is similar, but the constraint becomes severe. The p-value for obtaining a result favoring the normal hierarchy as much as the observed data is $0.43(0.02)$ assuming a normal (inverted) hierarchy and $\sin ^{2} \theta_{23}=0.6$.
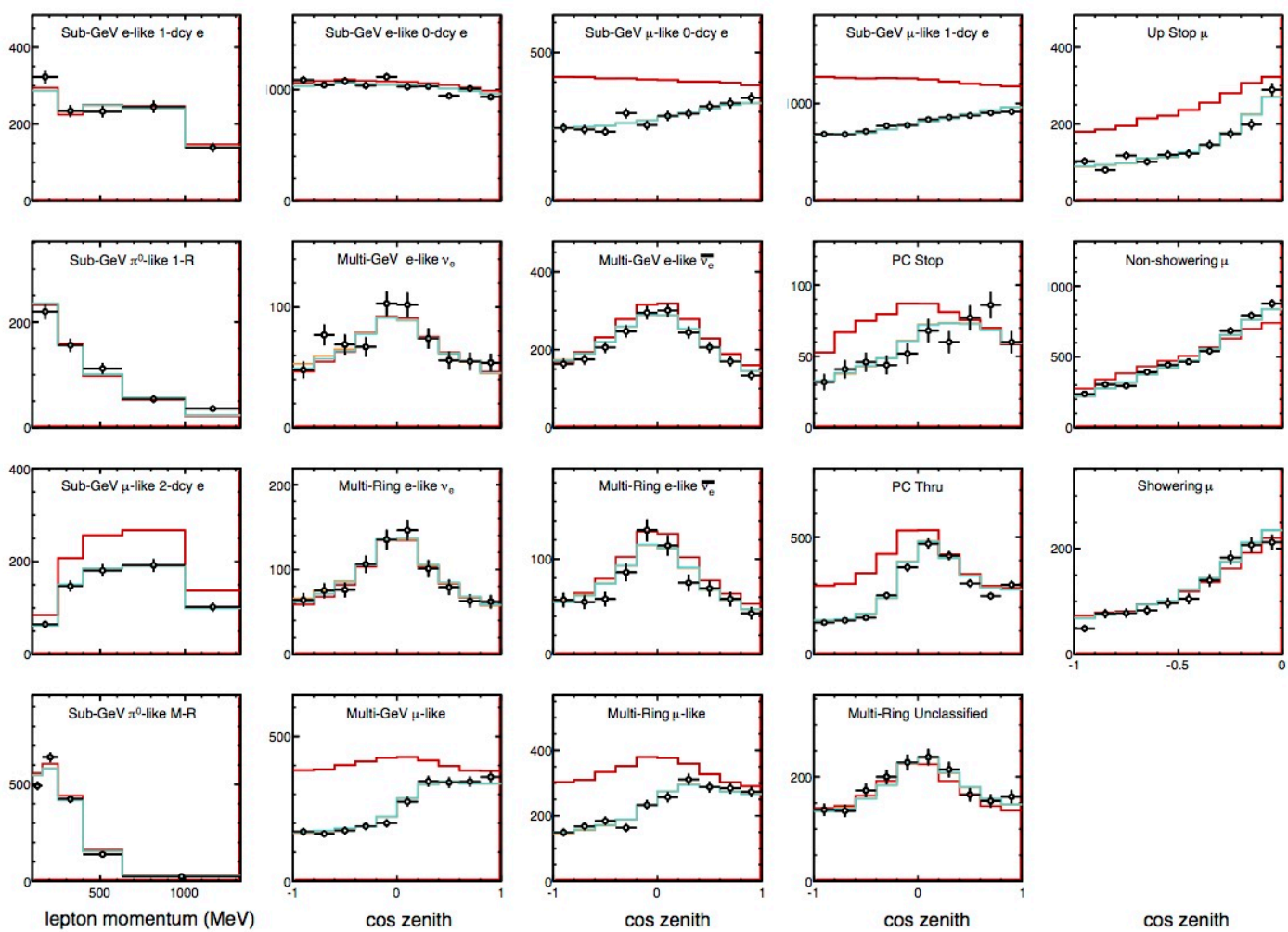

Figure 6: Momentum and zenith angle distribution of whole data category in SK-1 to IV overlaid with the expectation of with (green) / without (red) neutrino oscillation. 

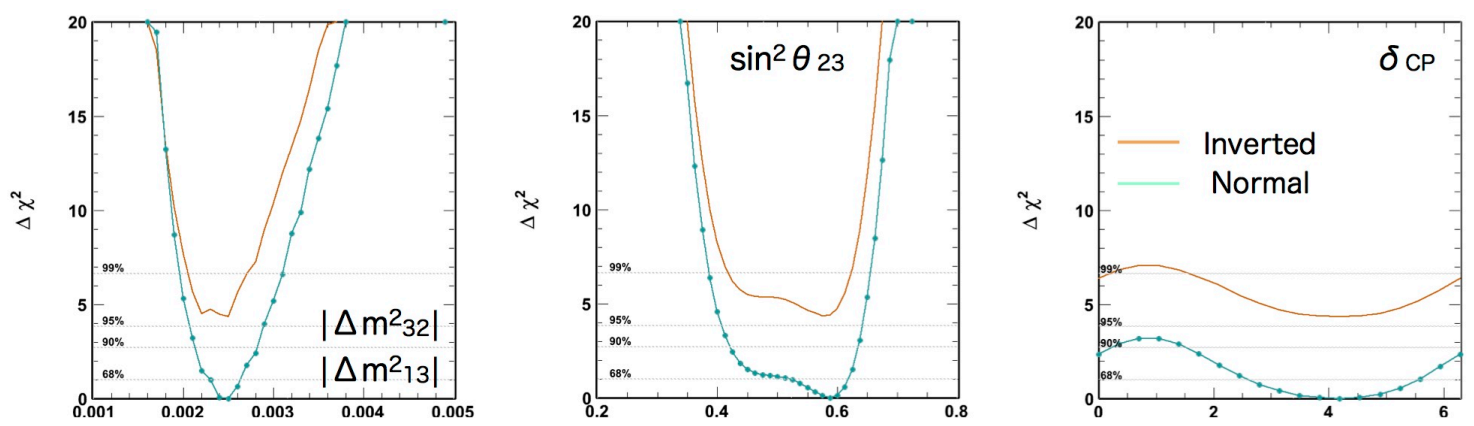

Figure 7: Chi-square distributions of $\Delta m_{32}^{2}, \sin ^{2} \theta_{23}, \delta_{C P}$ assuming normal and inverted hierarchy.

The analysis of the tau appearance by atmospheric neutrino oscillation is updated. Though it is hard to identify event by event for tau, but it can be seen statistically using neural network method. Figure 8$]$ shows the zenith angle distribution of multi-ring e-like events. Because of the tau appearance effect, the upward-going events should be increased compared to the case of no appearance, and the tau appearance significant is $4.6 \sigma$.

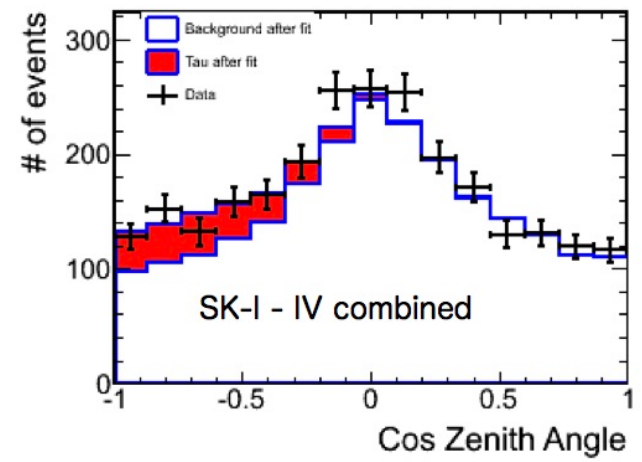

Figure 8: Zenith angle distribution of multi-ring elike events.

\section{Acknowledgments}

The author would like to thank the Super-Kamiokande collaboration for this work, also acknowledge the cooperation of the Kamioka Mining and Smelting Company. SK has been built and operated from funds provided by the Japanese Ministry of Education, Culture, Sports, Science and Technology, the U.S. Department of Energy, and the U.S. National Science Foundation.

\section{References}

[1] S. Fukuda et al., Nucl. Instrum. Meth. A501 (2003) 418.

[2] H. Nishino et al., Nucl. Instrum. Meth. A610 (2009) 710.

[3] K. Abe et al., Nucl. Instrum. Meth. A737C (2014) 253.

[4] M. Nakahata et al., Nucl. Instrum. Meth. A421 (1999) 113.

[5] E. Blaufuss et al., Nucl. Instrum. Meth. A458 (2001) 636.

[6] Y. Koshio, in proceedings of Neutrino 2014, AIPCP, (2015).

[7] E. Richard et al., Phys. Rev. D94 (2016) 052001.

[8] M. Honda et al., Phys. Rev. D83 (2011) 123001. 